\title{
Oncological resection, myasthenia gravis and staging as prognostic factors in thymic tumours: a Chilean case series
}

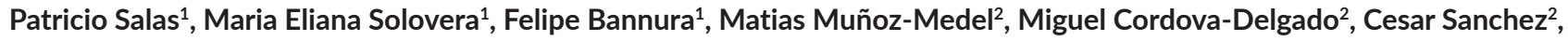 \\ Carolina lbañez ${ }^{2}$, Marcelo Garrido², Erica Koch², Francisco Acevedo², Sebastian Mondaca², Bruno Nervi², Jorge Madrid², Jose Peña², \\ Mauricio P Pinto², José Valbuena ${ }^{3}$ and Hector Galindo ${ }^{2}$ \\ ${ }^{1}$ Thoracic Surgery Section, Division of Surgery, Faculty of Medicine, Pontificia Universidad Catolica de Chile, Diagonal Paraguay 319, Santiago 8330032, \\ Chile \\ ${ }^{2}$ Department of Hematology and Oncology, Faculty of Medicine, Pontificia Universidad Catolica de Chile, Diagonal Paraguay 319, Santiago 8330032, Chile \\ ${ }^{3}$ Department of Pathology, Faculty of Medicine, Pontificia Universidad Catolica de Chile, Diagonal Paraguay 319, Santiago 8330032, Chile
}

\section{Abstract}

Background: Thymic epithelial tumours are rare and highly heterogeneous. Reports from the United States suggest an overall incidence of 0.15 per 100,000/year. In contrast, the incidence of these tumours in Latin America is largely unknown and reports are scarce, somewhat limited to case reports.

Methods: Herein, we report a series of 38 thymic tumours from a single institution, retrospectively incorporated into this study. Patient characteristics and outcomes including age, sex, stage, paraneoplastic syndromes, treatment regimens and the date of decease were obtained from medical records.

Results: Most cases in our series were females and young age ( $<50$ years old) and early stage by Masaoka-Koga or the Moran staging systems. Also, a 34\% of patients had myasthenia gravis (MG). Next, we analysed overall survival rates in our series and found that the quality of surgery (R0, R1 or R2), MG status and staging (Masaoka-Koga, Moran or TNM) were prognostic factors. Finally, we compared our data to larger thymic tumour series.

Conclusions: Overall, our study confirms complete surgical resection as the standard, most effective treatment for thymic epithelial tumours. Also, the Masaoka-Koga staging system remains as a reliable prognostic factor but also the Moran staging system should be considered for thymomas.

Keywords: thymic tumour, thymoma, myasthenia gravis, staging

\section{Introduction}

Thymic neoplasms are a rare and highly heterogeneous group of tumours. Reports from the United States indicate an overall incidence of 0.15 per 100,000/year [1]. Histologically, thymic tumours can be classified as thymomas, thymic carcinomas or
Correspondence to: Hector Galindo Email: investigacion.onco@uc.cl

ecancer 2021, 15:1201

https://doi.org/10.3332/ecancer.2021.1201

Published: 09/03/2021

Received: 06/11/2020

Publication costs for this article were supported by ecancer (UK Charity number 1176307).

Copyright: (c) the authors; licensee ecancermedicalscience. This is an Open Access article distributed under the terms of the Creative Commons Attribution License (http:// creativecommons.org/licenses/by/3.0), which permits unrestricted use, distribution, and reproduction in any medium, provided the original work is properly cited. 
neuroendocrine thymic tumours. These tumours are frequently associated with paraneoplastic diseases, among these myasthenia gravis (MG) is by far the most commonly reported. Indeed, most studies demonstrate that MG prevalence on thymoma patients ranges from $30 \%$ to $50 \%$ [2]. Although there is still no consensus on an official staging classification system for thymic malignancies [3], the system originally developed by Masaoka et al [4] and subsequently modified by Koga et al [5] remains as the most widely accepted. This system (hereafter called Masaoka-Koga) establishes four stages based on the local extent of the disease [6]. More recently, Moran et al [7] proposed a staging system based on pathological characteristics and specifically focused on thymomas and their invasiveness. Similarly, a number of histological classifications have been proposed for thymic tumours over the last decades; however, the World Health Organization histological classification system is the most accepted [8, 9]. Regarding treatment, the current gold standard for patients is complete resection surgery sometimes accompanied by radiation or chemotherapy [10].

As pointed above, thymic tumours are rare; however, their incidence in Latin America is largely undefined and the evidence is scarce and scattered, somewhat limited to case reports. Herein, we report a Chilean series of 38 thymic epithelial tumours from a single institution, most cases correspond to young patients ( $<50$-year-old) and early stage thymomas. We also report survival rates and compare our results to other published thymoma series.

\section{Materials and methods}

\section{Patients and ethics approval}

A total of 38 thymoma/thymic carcinoma patients were retrospectively incorporated into this study. Patients were diagnosed between December 1996 and April 2018. This study was approved by the Internal Review Board and Ethics committee at the Pontificia Universidad Catolica de Chile (approval \#171004005 dated on 7 November 2017). A waiver of consent was granted for deceased patients.

\section{Eligibility criteria and collected data}

Inclusion criteria for this study were: diagnosis of thymic epithelial tumour (either thymoma or thymic carcinoma), adult individuals (>18-yearold) at the time of diagnosis, availability of medical records including clinical management and follow-up and being able to understand, read and sign a written informed consent form. A minimum of 3 months of follow-up after surgical treatment was also considered as necessary for the incorporation of patients into this study. Collected data included: patient characteristics and outcomes such as age, sex, stage, paraneoplastic syndromes related to thymic malignancies, treatment regimens including chemotherapy, surgery and/or radiation therapy. The dates of decease were also obtained from medical records available at the Centro de Cancer in the Pontificia Universidad Catolica de Chile or the Red de Salud UC Christus in Santiago, Chile.

\section{Follow up, survival rates and statistics}

Last date of follow-up was 1 August 2019. Overall survival (OS) was defined as the time between diagnosis and death by any cause. Survival curves were estimated using the Kaplan-Meier method. Univariate associations between patient sex, age, stage, surgical resection and survival were analysed by Log-rank test. Statistical significance was set at $p<0.05$. Statistical analyses were performed using the $\mathrm{R}$ program software (version 3.5.1 R Development Core Team, R Foundation for Statistical Computing, Vienna, Austria), applying the 'survival' and 'survminer' packages.

\section{Results}

\section{Patients' characteristics}

Demographic and basic patient characteristics are summarised in Table 1. Briefly, most patients were female, younger than 50-year-old and early stage by Masaoka-Koga (50\% were stage I). Similarly, most thymomas in our series were categorised as stage 0-I (84\%) according to 
the staging system described by Moran et al [7]. As expected, the most frequent paraneoplastic symptom was MG (34\%). All patients had thymic resection surgery by sternotomy $(84 \%)$, video-assisted thoracic surgery (13\%) or by thoracotomy (8\%). Patients' subsets also received chemotherapy or radiation therapy, $37 \%$ or $21 \%$, respectively.

Table 1. Demographic, pathologic and treatment management characteristics of study population $(n=38)$.

\begin{tabular}{|c|c|c|c|c|c|}
\hline Characteristics & $n$ & $\%$ & Characteristics & $n$ & $\%$ \\
\hline \multicolumn{3}{|l|}{ Age at diagnosis } & \multicolumn{3}{|l|}{ WHO histologic classification } \\
\hline $25-49 y$ & 18 & 47 & A & 5 & 13 \\
\hline $50-70 y$ & 16 & 42 & $A B$ & 5 & 13 \\
\hline$>70 y$ & 4 & 11 & $\mathrm{~B} 1$ & 6 & 16 \\
\hline Sex & & & B2 & 9 & 24 \\
\hline Male & 14 & 37 & B3 & 6 & 16 \\
\hline Female & 24 & 63 & C & 7 & 18 \\
\hline \multicolumn{3}{|c|}{ Stage at diagnosis (Masaoka-Koga) } & \multicolumn{3}{|l|}{ Paraneoplastic symptoms } \\
\hline I & 19 & 50 & Myasthenia gravis & 13 & 34 \\
\hline Ila & 8 & 21 & Pure red cell aplasia & 2 & 5 \\
\hline $\mathrm{Ilb}$ & 3 & 8 & Nephrotic syndrome & 2 & 5 \\
\hline III & 5 & 13 & Agranulocytosis & 1 & 3 \\
\hline IVa & 3 & 8 & Surgical margins & & \\
\hline Stage at diagnosis (Moran) & & & RO & 33 & 87 \\
\hline 0 & 16 & 59 & R1 & 2 & 5 \\
\hline I & 7 & 26 & $\mathrm{R} 2$ & 3 & 8 \\
\hline II & 3 & 11 & Resection of regional lymph nodes & & \\
\hline III & 1 & 4 & Yes & 21 & 55 \\
\hline \multicolumn{3}{|c|}{ Primary tumor size (AJCC $8^{\text {th }}$ Edition) } & No & 14 & 37 \\
\hline T1a & 29 & 76 & NA & 3 & 8 \\
\hline $\mathrm{T} 2$ & 1 & 3 & Chemotherapy & & \\
\hline T3 & 7 & 18 & No & 24 & 63 \\
\hline T4 & 1 & 3 & Yes & 5 & 13 \\
\hline \multicolumn{3}{|c|}{ Regional lymph nodes (AJCC $8^{\text {th }}$ Edition) } & NA & 9 & 24 \\
\hline NO & 37 & 97 & \multicolumn{3}{|l|}{ Radiation therapy } \\
\hline N1 & 1 & 3 & No & 22 & 58 \\
\hline \multicolumn{3}{|c|}{ Distant metastasis (AJCC $8^{\text {th }}$ Edition) } & Yes & 8 & 21 \\
\hline MO & 34 & 89 & NA & 8 & 21 \\
\hline M1a & 4 & 11 & \multicolumn{3}{|l|}{ Thymic epithelial tumor histotype } \\
\hline \multicolumn{3}{|c|}{ TNM stage at diagnosis (AJCC $8^{\text {th }}$ Edition) } & Thymoma & 31 & 82 \\
\hline I & 28 & 74 & Thymic carcinoma & 6 & 16 \\
\hline II & 1 & 3 & Mixed $†$ & 1 & 3 \\
\hline IIla & 4 & 11 & \multicolumn{3}{|l|}{ Surgery type } \\
\hline IVa & 5 & 13 & Sternotomy & 32 & 84 \\
\hline & & & VATS & 5 & 13 \\
\hline & & & Thoracotomy & 1 & 3 \\
\hline
\end{tabular}

VATS, video-assisted thoracic surgery; NA, not available. † One case evidenced both thymoma and thymic carcinoma cells during histologic analysis. 


\section{Survival rates and current thymoma series versus other cohorts}

OS rates for the entire series are shown in Figure 1a. As expected, tumour stage at initial diagnosis had a significant impact on OS ( $p<0.0001$, Figure 1b). Five-year OS was $89 \%, 100 \%, 80 \%$ and $0 \%$ for Masaoka stage I, II, III, IV, respectively. All patients underwent surgical resection with curative intent. Negative resection margins with pathologically confirmed (R0) were achieved in 33 (87\%) patients. Otherwise, three (8\%) and two (5\%) patients had microscopic residual disease (R1) and gross residual disease (R2). Also as expected, patients with an optimal surgery (RO) had better OS versus suboptimal (R1 or R2) counterparts ( $p<0.0001$, Figure 1c). Five-year OS was $94 \%, 50 \%$ and $0 \%$ for R0, R1 and R2 resection, respectively. Patients that were MG+ had better OS rates versus MG- counterparts ( $p<0.05$, Figure $1 d$ ). Then, we analysed OS in the subset of thymomas $(n=32)$ in our series according to the Moran et al [7] staging system and found a significant impact on survival (Figure 1e; $p$ 0.005). Lastly, as shown in Table 2, we compared OS rates by Masaoka-Koga stage in our series against other previously published cohorts from Japan [4], Canada [11], the Netherlands [12] and China [13]. Patient follow-up and other symptoms are summarized in Supplementary Table S1.

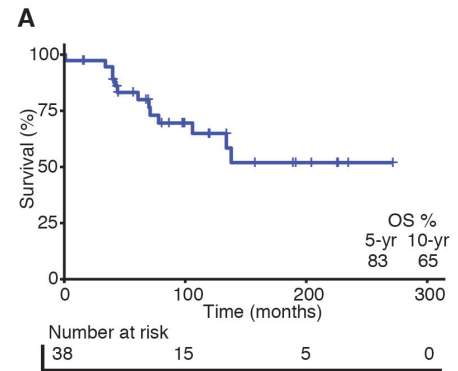

C
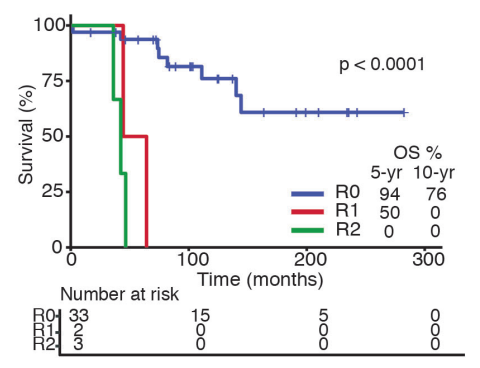

E

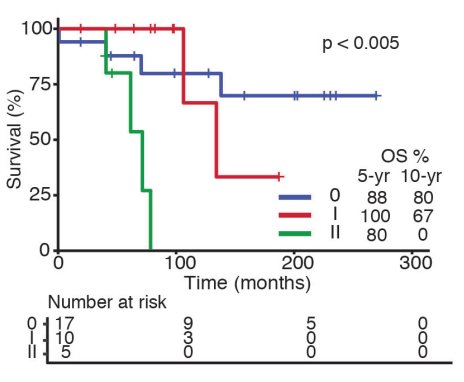

B

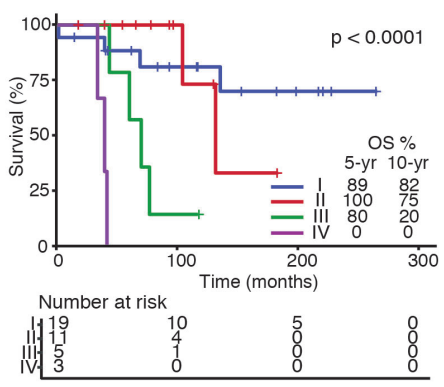

D

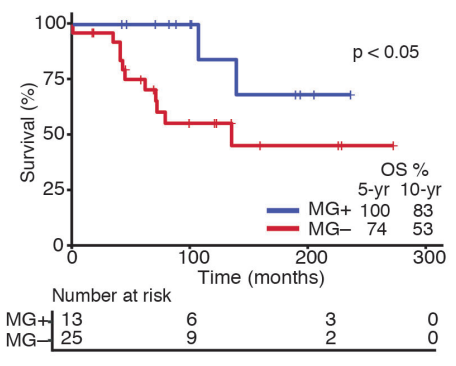

$\mathbf{F}$

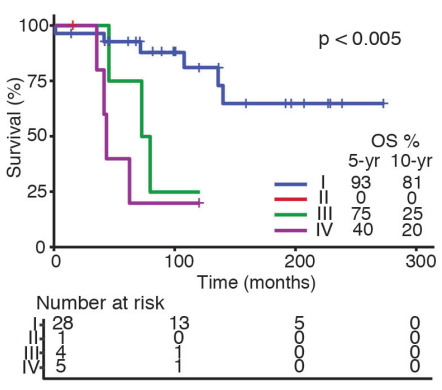

Figure 1. Survival curves in Chilean thymic tumour series. (a): OS for the entire series $(n=38)$. (b): OS by Masaoka-Koga staging system at presentation. (c): OS by status of surgical margins at oncological resection. (d): OS by presence or absence of MG at diagnosis. (e): OS by Moran staging at diagnosis. (f): OS by TNM staging at diagnosis. Abbreviations: OS, overall survival; yr, Year; MG, Myasthenia gravis. 
Table 2. Survival rates comparison of thymoma series by Masaoka-Koga staging system.

\begin{tabular}{|c|c|c|c|c|c|c|c|c|c|}
\hline \multirow{2}{*}{ Stage } & \multicolumn{3}{|c|}{ Chilean thymomas } & \multicolumn{2}{|c|}{ Masaoka et al [4] } & \multicolumn{2}{|c|}{ Mariano et al [11] } & \multirow{2}{*}{$\begin{array}{c}\text { d de Jong et al [12] } \\
5-y r \text { OS\% }\end{array}$} & \multirow{2}{*}{$\begin{array}{c}\text { Zhu et al [13] } \\
5-y r \text { OS\% }\end{array}$} \\
\hline & $n$ & $5-y r$ OS\% & $10-y r$ OS\% & 5-yr OS\% & $10-y r$ OS\% & 5-yr OS\% & $10-y r$ OS\% & & \\
\hline 1 & 19 & 89 & 82 & 96 & 67 & 93 & 93 & 83 & 96 \\
\hline II & 11 & 100 & 75 & 86 & 60 & 89 & 87 & 88 & 89 \\
\hline III & 5 & 80 & 20 & 70 & 58 & 75 & 62 & 57 & 59 \\
\hline IV & 3 & 0 & 0 & 50 & 0 & 43 & 29 & 56 & 50 \\
\hline
\end{tabular}

yr, Year; OS, Overall survival

\section{Discussion}

Thymic tumours are infrequent neoplasms. Epidemiological studies in the US indicate an incidence of 0.15 cases per 100,000/year [1]. In contrast, thymic tumour incidence in Latin America is largely unknown. Furthermore, regional studies on thymic tumours/thymomas are extremely scarce. Two retrospective single-institution studies in Mexico City have reported 64 [14] and 25 [15] patients, respectively. The first study reports a 10 -year database in which $54.7 \%$ were males and average age (at diagnosis) was 51.4 years. $28 \%$ of patients were MG+. The second study reports clinical characteristics of patients recruited in the period January 2005-December 2016, although these data are unpublished, they were presented at the International Association for the Study of Lung Cancer 18th World Conference on Lung Cancer [15]. In Chile, a recent study reported survival rates and basic characteristics of 65 thymic tumour patients [16]; however, this report does not provide information on long-term OS rates (5- or 10-year). In general, thymoma series demonstrate a predominance of females over males, and diagnosis at an early stage. Accordingly, our study found that most patients (63\%) were female and early stage (50\% were stage I). As expected, OS rates in our study correlated with tumour stage by Masaoka-Koga. This is also in line with published larger thymoma series (Table 2) that demonstrate lower 5- and 10-year OS at increasing tumour stages. Over the last century, a variety of staging systems have been proposed for thymic tumours [3]; however, the Masaoka-Koga staging system remains as the preferred choice in the literature. Within this context, a recent study by Weissferdt et al [17] proposes a simplified staging system for thymomas, originally described by Moran et al [7]. Authors used a large cohort ( $n=1,470$ cases) and demonstrate a significant correlation with clinical outcomes [17]. Accordingly, in our data, we found a significant correlation to OS using this staging system; however, this was limited to the subset of thymomas (Figure $1 \mathrm{e}$ ). Also as expected, the most frequent paraneoplastic symptom was MG (34\%). Interestingly, our data suggest that MG+ patients had better OS versus MG- counterparts (Figure 1d). Indeed, previous studies have described a better OS in MG+ thymomas [18]. However, subsequent stageadjusted studies indicated no differences in survival between MG+ and MG- thymomas [8, 19, 20]. More recently, a large retrospective study found that MG+ had a slight protective effect on OS among thymoma patients. Once again, this association could not be confirmed by a multivariate analysis. Similarly, the abovementioned retrospective study in Mexico found a trend towards better OS rates in MG+ that did not reach statistical significance. Although a potential link between MG+ and better OS in these patients remains controversial, authors have speculated that the correlation between MG+ and early Masaoka-Koga stages could explain this association [9]. Furthermore, the same study also demonstrated that a higher Masaoka-Koga stage correlated with non-MG status. Evidently, our findings warrant further investigation and should be confirmed by larger series.

\section{Conclusion}

Overall, our study confirms complete surgical resection as the standard, most effective treatment for thymic tumours. Similarly, the MasaokaKoga staging system remains as a reliable prognostic factor for these tumours. In the same way, the Moran staging system for thymomas demonstrates a better stratification for this subset of thymic tumours, especially at 10-years and further. Unexpectedly, our data report a statistically significant difference in OS regarding MG status, with better rates of OS at 5 and 10 years for MG+ patients. Finally, this study has a number of limitations: first and foremost, the number of cases is relatively small compared to other published series (Table 2), this is partially explained by the total Chilean population ( 17.5 million) and the low incidence rates of these tumours. Secondly, as pointed out, our data were retrospectively obtained at a single institution which may represent a registration bias that may limit the scope of our findings. 


\section{Availability of data and materials}

The data used and analysed on this study are available from the corresponding author on reasonabe request.

\section{Contributions}

PS and MES conceived the present study. MES and MMM collected the data. MCD, MMM and MPP analysed and interpreted the data. MPP drafted the manuscript. PS, MES, FB, CS, CI, MG, EK, FA, SM, BN, JM, JP, JV and HG critically revised the manuscript. All authors read and approved the final version of the manuscript.

\section{Ethics declaration}

\section{Ethics approval and consent to participate}

Study and ethics approval were obtained by the Internal Review Board and Ethics committee at the Pontificia Universidad Catolica de Chile (approval \#171004005 dated on 7 November, 2017).

\section{Funding statement}

None.

\section{Competing interests}

The authors declare that they have no competing interests.

\section{References}

1. Engels EA and Pfeiffer RM (2003) Malignant thymoma in the United States: demographic patterns in incidence and associations with subsequent malignancies Int J Cancer 105 546-551 https://doi.org/10.1002/ijc.11099 PMID: 12712448

2. Rashid OM, Cassano AD, and Takabe K (2013) Thymic neoplasm: a rare disease with a complex clinical presentation J Thorac Dis 5 173-183 PMID: 23585946 PMCID: 3621939

3. Roden AC (2017) Evolution of classification of thymic epithelial tumors in the era of Dr Thomas V. Colby Arch Pathol Lab Med 141 232-246 https://doi.org/10.5858/arpa.2016-0057-RA PMID: 28134578

4. Masaoka A, Monden Y, and Nakahara K, et al (1981) Follow-up study of thymomas with special reference to their clinical stages Cancer 48 2485-2492 https://doi.org/10.1002/1097-0142(19811201)48:11<2485::AID-CNCR2820481123>3.0.CO;2-R PMID: 7296496

5. Koga K, Matsuno Y, and Noguchi M, et al (2008) A review of 79 thymomas: modification of staging system and reappraisal of conventional division into invasive and non-invasive thymoma Pathol Int 44 359-367 https://doi.org/10.1111/j.1440-1827.1994.tb02936.x

6. Masaoka A (2010) Staging system of thymoma J Thorac Oncol 5 S304-S312 https://doi.org/10.1097/JTO.0b013e3181f20c05 PMID: 20859124 
7. Moran CA, Walsh G, and Suster S, et al (2012) Thymomas Il: a clinicopathologic correlation of 250 cases with a proposed staging system with emphasis on pathologic assessment Am J Clin Pathol 137 451-461 https://doi.org/10.1309/AJCP36ALGUZWOSEA PMID: 22338058

8. Okumura M, Ohta M, and Tateyama H, et al (2002) The World Health Organization histologic classification system reflects the oncologic behavior of thymoma Cancer 94 624-632 https://doi.org/10.1002/cncr.10226 PMID: 11857293

9. Ruffini E, Filosso PL, and Mossetti C, et al (2011). Thymoma: inter-relationships among World Health Organization histology, Masaoka staging and myasthenia gravis and their independent prognostic significance: a single-centre experience Eur J Cardio-Thoracic Surg 40 146-153 https://doi.org/10.1016/j.ejcts.2010.09.042

10. Ruffini E and Venuta F (2014) Management of thymic tumors: a European perspective J Thorac Dis 6 S228-S237 PMID: 24868441 PMCID: 4032964

11. Mariano C, lonescu DN, and Cheung WY, et al (2013). Thymoma: a population-based study of the management and outcomes for the province of British Columbia J Thorac Oncol 8 109-117 https://doi.org/10.1097/JTO.0b013e318276241c

12. de Jong WK, Blaauwgeers JLG, and Schaapveld M, et al (2008) Thymic epithelial tumours: a population-based study of the incidence, diagnostic procedures and therapy Eur J Cancer 44 123-130 https://doi.org/10.1016/j.ejca.2007.11.004

13. Zhu L, Zhang J, and Marx A, et al (2016). Clinicopathological analysis of 241 thymic epithelial tumors-experience in the Shanghai Chest Hospital from 1997-2004 J Thorac Dis 8 718-726 https://doi.org/10.21037/jtd.2016.03.24 PMID: 27114840 PMCID: 4824732

14. Cacho-Díaz B, Salmerón-Moreno K, and Lorenzana-Mendoza NA, et al (2018) Myasthenia gravis as a prognostic marker in patients with thymoma J Thorac Dis 10 2842-2848 https://doi.org/10.21037/jtd.2018.04.95 PMID: 29997948 PMCID: 6006064

15. López Saucedo R, Jiménez Fuentes E, and De La Garza J, et al (2017) P1.17-008 clinical and oncological outcomes on resected thymomas over a decade at the National Cancer Institute at Mexico City J Thorac Oncol 12 S2063-S2064 https://doi.org/10.1016/j. jtho.2017.09.1090

16. Salinas M, Liessi M, and Vallejo P, et al (2019) Survival in a Chilean Cohort of thymic tumors J Cancer Ther 10 $510-517$ https://doi. org/10.4236/jct.2019.107042

17. Weissferdt A, Kalhor N, and Bishop JA, et al (2018) Thymoma: a clinicopathological correlation of 1470 cases Hum Pathol 73 7-15 https://doi.org/10.1016/j.humpath.2017.08.018

18. Margaritora S, Cesario A, and Cusumano G, et al (2010) Thirty-five-year follow-up analysis of clinical and pathologic outcomes of thymoma surgery Ann Thorac Surg 89 245-252 https://doi.org/10.1016/j.athoracsur.2009.08.074 PMID: 20103246

19. Kondo K and Monden Y (2003) Therapy for thymic epithelial tumors: a clinical study of 1,320 patients from Japan Ann Thorac Surg 76 878-884 https://doi.org/10.1016/S0003-4975(03)00555-1 PMID: 12963221

20. Okereke IC, Kesler KA, and Morad MH, et al (2010) Prognostic indicators after surgery for thymoma Ann Thorac Surg 89 1071-1079 https://doi.org/10.1016/j.athoracsur.2010.01.026 PMID: 20338309 


\section{Supplementary information}

Supplementary Table S1. Onset symptoms, follow-up and survival of study population $(n=38)$.

\begin{tabular}{|c|c|c|}
\hline & $n$ & $\%$ \\
\hline \multicolumn{3}{|l|}{ Presenting symptoms } \\
\hline Arterial hypertension & 7 & 18 \\
\hline Diabetes mellitus & 1 & 3 \\
\hline Sjögren syndrome & 2 & 5 \\
\hline Dyslipidaemia & 5 & 13 \\
\hline SLE & 1 & 3 \\
\hline Cancer $^{\mathrm{a}}$ & 6 & 16 \\
\hline Chest pain & 6 & 16 \\
\hline Hypothyroidism & 4 & 11 \\
\hline Hyperparathyroidism & 1 & 3 \\
\hline Diplopia & 3 & 8 \\
\hline SVC syndrome & 1 & 3 \\
\hline Asymptomatic & 5 & 13 \\
\hline \multicolumn{3}{|l|}{ Follow-up and survival } \\
\hline Alive & 25 & 66 \\
\hline Median follow-up (range), months & \multicolumn{2}{|c|}{$83(1-271)$} \\
\hline
\end{tabular}

SLE, Systemic lupus erythematosus; SVC, Superior vena cava aOne case of thymoma had a concomitant finding of early-stage lung adenocarcinoma 